\title{
Corollary Discharge Inhibition of Ascending Auditory Neurons in the Stridulating Cricket
}

\author{
James F. A. Poulet and Berthold Hedwig \\ Department of Zoology, University of Cambridge, Cambridge CB2 3EJ, United Kingdom
}

Acoustically communicating animals are able to process external acoustic stimuli despite generating intense sounds during vocalization. We have examined how the crickets' ascending auditory pathway copes with self-generated, intense auditory signals (chirps) during singing (stridulation). We made intracellular recordings from two identified ascending auditory interneurons, ascending neuron 1 (AN1) and ascending neuron 2 (AN2), during pharmacologically elicited sonorous (two-winged), silent (one-winged), and fictive (isolated CNS) stridulation.

During sonorous chirps, AN1 responded with bursts of spikes, whereas AN2 was inhibited and rarely spiked. Low-amplitude hyperpolarizing potentials were recorded in AN1 and AN2 during silent chirps. The potentials were also present during fictive chirps. Therefore, they were the result of a centrally generated corollary discharge from the stridulatory motor network. The spiking response of AN1 and AN2 to acoustic stimuli was inhibited during silent and fictive chirps. The maximum period of inhibition occurred in phase with the maximum spiking response to self-generated sound in a sonorously stridulating cricket. In some experiments (30\%) depolarizing potentials were recorded during silent chirps. Reafferent feedback elicited by wing movement was probably responsible for the depolarizing potentials.

In addition, two other sources of inhibition were present in AN1: (1) IPSPs were elicited by stimulation with $12.5 \mathrm{kHz}$ stimuli and (2) a long-lasting hyperpolarization followed spiking responses to $4.5 \mathrm{kHz}$ stimuli. The hyperpolarization desensitized the response of AN1 to subsequent quieter stimuli. Therefore, the corollary discharge will reduce desensitization by suppressing the response of AN1 to selfgenerated sounds.

Key words: corollary discharge; efference copy; stridulation; presynaptic inhibition; postsynaptic inhibition; ascending neuron 1; ascending neuron 2

\section{Introduction}

Sensory processing is generally studied by presenting stimuli to resting or anesthetized animals. However, in their natural environment, an animal's sensory systems will respond both to external sensory information and to sensory information generated as a byproduct of its behavior. To cope with intense self-generated, or reafferent (von Holst and Mittelstaedt, 1950), sensory information many animals reduce the sensitivity of their sensory pathway while generating reafferent information (Murphey and Palka, 1974; Zaretsky and Rowell, 1979; Bell, 1981, 1982; Guthrie et al., 1983; Sillar and Skorupski, 1986; Sillar and Roberts, 1988; Gossard et al., 1991; Wolf and Burrows, 1995; El Manira et al., 1996; Blakemore et al., 1998; Bodznick et al., 1999). This can be achieved by a modulation in the biophysical sensitivity of the peripheral sense organ (Suga and Jen, 1975; Borg and Counter, 1989; Narins, 1992; Hennig et al., 1994) or by neural inhibition of the central sensory pathway. If the inhibition is generated within the nervous system it is termed an efference copy (von Holst and Mittelstaedt, 1950) or corollary discharge (Sperry, 1950). In previous experiments we have established that during stridulation the cricket's peripheral auditory system remains fully sensitive (Poulet and Hedwig, 2001), but its central auditory system is

Received Dec. 6, 2002; revised Feb. 3, 2003; accepted Feb. 21, 2003.

This work was supported by a Biotechnology and Biological Sciences Research Council studentship and grants from the Royal Society and the Wellcome Trust.

Correspondence should be addressed to Dr. James Poulet, Department of Zoology, University of Cambridge, Cambridge CB2 3EJ, UK. E-mail: jfap2@cam.ac.uk.

Copyright $\odot 2003$ Society for Neuroscience $\quad$ 0270-6474/03/234717-09\$15.00/0 inhibited by a corollary discharge (Poulet and Hedwig, 2002, 2003). The inhibition reduces the neuronal response to the cricket's own sound and helps maintain the responsiveness of a local auditory interneuron, the omega 1 neuron $(\mathrm{ON} 1)$, to external sounds during stridulation (Poulet and Hedwig, 2002, 2003).

In the cricket's auditory pathway, information ascends from the prothoracic ganglion to the brain via ascending auditory interneurons. Ascending neuron 1 (AN1) responds best to $4.5 \mathrm{kHz}$, the carrier frequency of calling song, and is thought to process conspecific sounds (Boyan, 1980; Wohlers and Huber, 1982; Stumpner et al., 1995). Ascending neuron 2 (AN2), on the other hand, is inhibited by $4.5 \mathrm{kHz}$ and responds best to higher frequencies (10-30 kHz) (Wohlers and Huber, 1978, 1982; Boyan, 1980; Nolen and Hoy, 1987). Therefore, it is thought to play a role in high-frequency courtship song recognition (Wohlers and $\mathrm{Hu}-$ ber, 1982) and to act as a bat detector during flight (Nolen and Hoy, 1983). We examined how AN1 and AN2 cope with intense reafferent stimulation and analyzed their activity and response properties during pharmacologically elicited sonorous, silent, and fictive stridulation. Therefore, this study characterizes what information is transmitted to the brain while the animals are singing.

\section{Materials and Methods}

Details of all methods used have been described previously (Poulet and Hedwig, 2003). Briefly, experiments were performed on several hundred adult male Gryllus bimaculatus, and data were collected from 84 of these animals. Stridulation was elicited by injection of the acetylcholinesterase inhibitor physostigmine salicylate $\left(10^{-2} \mathrm{~mol}\right.$ per liter in insect saline) 


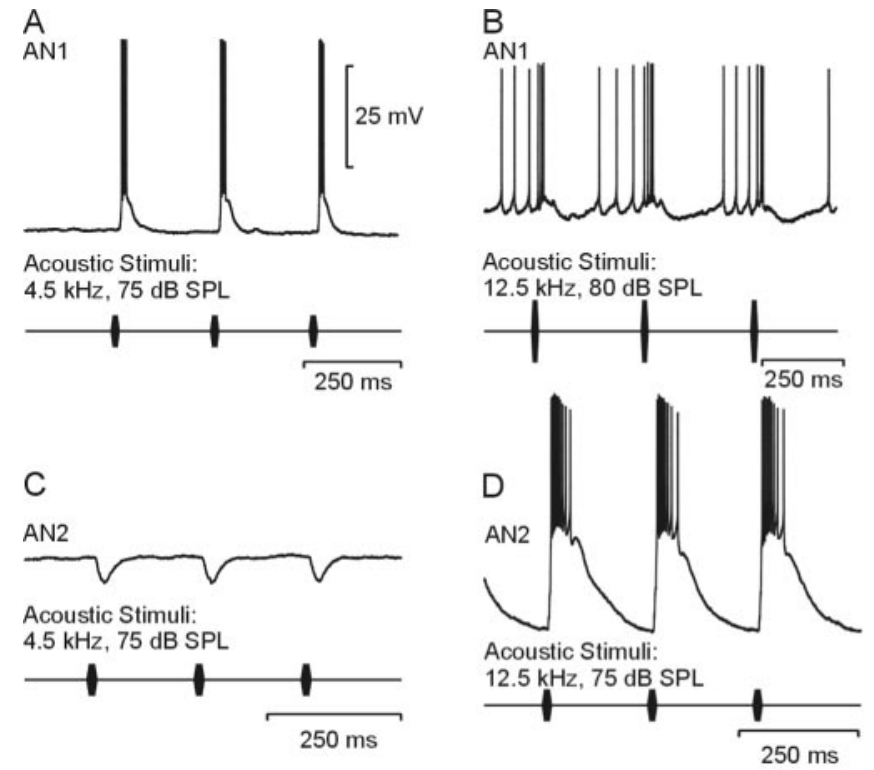

Figure 1. Responses of AN1 and AN2 to 4.5 and $12.5 \mathrm{kHz}$ stimulation. Acoustic stimuli of 4.5 kHz elicit a burst of $\sim 4$ spikes in AN1 $(A)$, whereas at $80 \mathrm{dBSPL}, 12.5 \mathrm{kHz}$ stimuli cause a mix of excitation and inhibition in AN1 (B). Acoustic stimuli of $75 \mathrm{~dB} \mathrm{SPL}, 4.5 \mathrm{kHz}$ causes IPSPs in AN2 (C), whereas $75 \mathrm{dBSPL}, 12.5 \mathrm{kHz}$ stimuli elicit a burst of spikes (D). AN1, Intracellular recording of AN1; AN2, intracellular recording of AN2;Acoustic Stimuli, sound pulses.

into the anterior protocerebrum of the brain (Otto, 1978; details in Wenzel and Hedwig, 1999). Intracellular recordings of AN1 and AN2 were made from their dendritic region in the auditory neuropil of the prothoracic ganglion using thick-walled glass micropipettes. All neurons were stained with Lucifer yellow and identified under an ultraviolet fluorescence microscope. Separate animals were used for each of the preparations (sonorous/silent/fictive stridulation). Therefore, recordings of $\mathrm{AN} 1$ and AN2 were normally made from different animals. In fictively singing crickets, extracellular recordings of motor activity were made from nerve $3 \mathrm{~A}$ of the mesothoracic ganglion.

Repetitive acoustic stimuli were presented with a short duration (8 $\mathrm{msec}$ ) and period $(15 \mathrm{msec})$ at $75 \mathrm{~dB}$ sound pressure level (SPL) relative to $20 \mu \mathrm{Pa}$ root mean square (RMS) to obtain as many data points as possible throughout the chirp and chirp interval. To mimic cricket calling song, stimuli had the natural syllable duration $(21 \mathrm{msec})$, period (42 msec), intensity (100 dB SPL RMS), and frequency ( $4.5 \mathrm{kHz})$. Stimuli presented to AN2 during silent stridulation had a duration of $21 \mathrm{msec}$, an intensity of $75 \mathrm{~dB}$ SPL (RMS), and a period of $250 \mathrm{msec}$. The relatively long period gave AN2 sufficient recovery time. Acoustic stimuli had a carrier frequency of 4.5 or $12.5 \mathrm{kHz}$. All analysis was done using the software packages Neurolab (Knepper and Hedwig, 1997) and Microsoft Excel 1997 (Microsoft, Seattle, WA). The number of neuronal recordings of a particular physiological effect that was quantitatively analyzed was sometimes smaller than the total number of recordings of the effect, because occasionally the section of data were too short to allow a full analysis. All spike frequencies are instantaneous spike frequencies calculated from the interspike intervals of consecutive spikes.

\section{Results}

\section{Responses of AN1 and AN2 to external acoustic stimuli in resting crickets}

The dendritic branches and ascending axons of AN1 and AN2 are located contralateral to their soma in the auditory neuropil of the prothoracic ganglion, in which they make monosynaptic connections to auditory afferents (Wohlers and Huber, 1982; Hennig, 1988). AN1 is strongly excited by $4.5 \mathrm{kHz}$ (Wohlers and Huber, 1982) (Fig. 1A), whereas stimulation with $75 \mathrm{~dB}$ SPL, $12.5 \mathrm{kHz}$ acoustic stimuli elicited IPSPs $(n=8)$. At higher intensities 12.5 $\mathrm{kHz}$ stimuli elicited a combination of excitatory and inhibitory responses in AN1 (Fig. 1B). In four recordings, subthreshold EPSPs were recorded in response to $12.5 \mathrm{kHz}, 75 \mathrm{~dB}$ SPL acoustic stimuli. In AN2, $4.5 \mathrm{kHz}$ stimuli generally elicited IPSPs $(n=10)$ (Nolen and Hoy, 1987) (Fig. 1C). In four other crickets, the response to $4.5 \mathrm{kHz}$ was a mix of inhibition and excitation. Occasionally, no IPSPs were recorded in response to $4.5 \mathrm{kHz}$ stimuli $(n=4)$; in these cases, AN2 responded with EPSPs and sometimes spikes. AN2 responded to $12.5 \mathrm{kHz}$ acoustic stimuli with a burst of spikes (Wohlers and Huber, 1982) (Fig. 1D).

\section{Responses of AN1 and AN2 during sonorous stridulation}

Stridulating male Gryllus bimaculatus rub their forewings together to generate $100 \mathrm{~dB}$ SPL sound pulses, generally termed syllables, with a carrier frequency of $4.5 \mathrm{kHz}$; these are arranged into chirps (Fig. 2Ai). During sonorous chirps, AN1 was rhythmically excited and produced bursts of spikes in phase with the syllables (Fig. $2 A$, asterisks). Wing opening at the start of a chirp produced a quiet sound (Fig. $2 A i$, arrow 1 ) that caused a depolarizing potential in AN1 (Fig. $2 A i$, arrow 2) and sometimes a spike. Thereafter, AN1 produced bursts of spikes in response to the louder syllables generated during wing closing (Fig. $2 \mathrm{Ai}$, asterisk). Quantitative evaluation of the response of AN1 during sonorous stridulation shows the peristimulus time (PST) histogram overlaid with the spike frequency (Fig. 2Aii, top traces) along with the average wing movement (Fig. 2 Aii, middle trace) and rectified sound recording (Fig. 2 Aii, bottom trace). The spiking response correlates with the sound intensity, with the loudest syllable, generated during wing closing (Fig. 2 Aii, asterisk), causing the strongest spiking response, during wing opening (Fig. 2 Aii, dashed line). On average, AN1 reached a maximum spike frequency of $183 \pm 25 \mathrm{~Hz}$ during sonorous chirps (mean \pm SEM, $n=6$ crickets).

In contrast to the response of AN1, AN2 was inhibited and rarely spiked during sonorous chirps (Fig. $2 B$ ) ( $n=5$ crickets). At the start of the chirp an IPSP occurred at the transition between wing opening and closing (Fig. $2 \mathrm{Bi}$, solid vertical line). This was generated in response to the quiet sound produced during wing opening (Fig. $2 \mathrm{Bi}$, arrow 3 ). Thereafter, IPSPs occurred in phase with the opening movement (Fig. $2 \mathrm{Bi}$, dashed line), which followed the loud sounds produced during wing closing (Fig. $2 \mathrm{Bi}$, arrow 4). Between each IPSP the membrane potential of AN2 repolarized (Fig. $2 \mathrm{Bi}$, asterisk); however, the repolarization was so rapid that it seems likely that this was in part attributable to an excitatory input during singing. High-intensity 4.5 $\mathrm{kHz}$ signals or high-frequency harmonics of the calling song could have excited AN2. The quantitative evaluation of the response of AN2 during sonorous stridulation confirmed that few spikes were generated during sonorous chirps (Fig. 2 Bii). The responses of AN1 and AN2 during stridulation were similar to their responses to $4.5 \mathrm{kHz}$ acoustic stimuli at rest (Fig. 1). Therefore, we suspected that these neurons were responding to reafferent sound. To test this, we recorded their responses during "silent" stridulation in crickets with one wing removed.

\section{Responses of AN1 and AN2 during silent stridulation}

The spikes in AN1 and high-amplitude IPSPs in AN2 recorded during sonorous stridulation were absent during silent stridulation. Therefore, these responses were a reaction to self-generated sounds. We recorded small hyperpolarizing potentials during silent chirps in nine recordings of AN1 (Fig. 3Ai) and nine recordings of AN2 (Fig. 3Bi). The hyperpolarizations began just after the start of wing closing (Fig. 3 Aii, Bii, dashed lines) and reached a maximum during the consecutive wing opening 


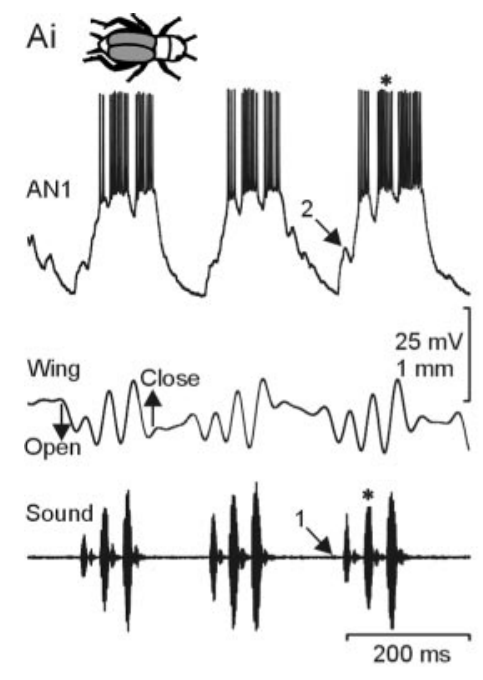

$\mathrm{Bi}$
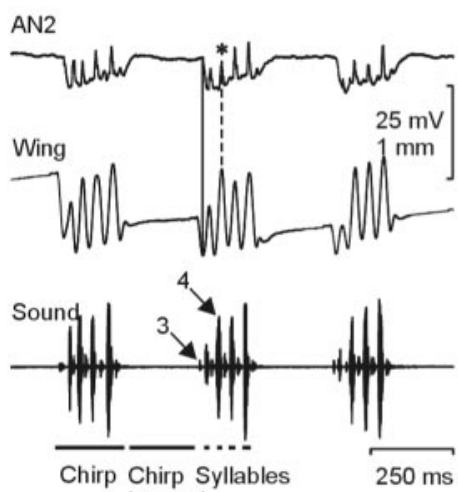

Interval

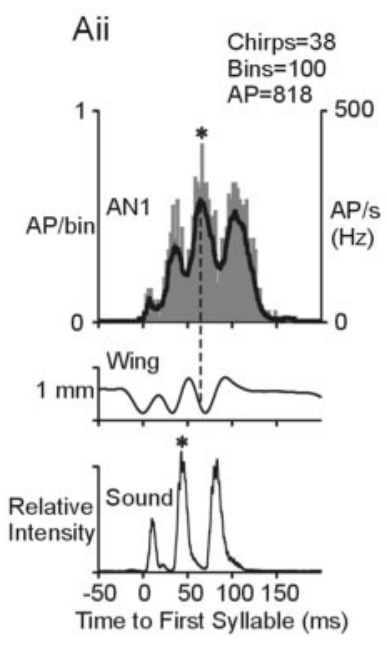

Bii
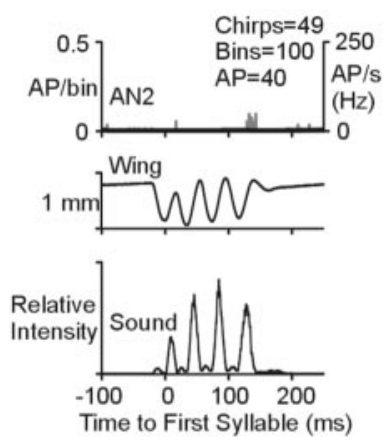

Figure 2. Activity of auditory neurons during sonorous singing. Ai, AN1 responded to the loud syllables (asterisk) produced during the chirp. The quieter sounds (arrow 1) caused by wing opening generated a depolarizing potential (arrow 2), which occasionally elicited a spike. Wing opening and closing are marked at the side of the wing recording. Aii, The PST histogram and the overlaid, averaged spike frequency (top), averaged wing movement (middle), and rectified sound recording (bottom) show that the response of AN1 is in phase with sound production. The peak response of AN1 occurs during the closing wing movements and is indicated by a dashed line. Bi, AN2 was inhibited during sonorous chirps, an IPSP was generated in response to the initial quiet sound produced by opening wing movement (arrow 3 ) and in response to each loud sound generated during wing closing (arrow 4). Between each IPSP, AN2 rapidly repolarized (asterisk). A chirp, chirp interval, and syllables are marked below the recording. Bii, The PST histogram and overlaid spike frequency show that AN2 rarely spiked during sonorous stridulation. Crickets above the figures symbolize two-winged, one-winged, or fictive singing with or without ears (see Figs. 3 to 10). Wing, Stridulatory wing movements; Sound, microphone recording; AP, Action potential.

(Fig. 3Aii,Bii, solid vertical lines). On average, the maximum amplitude of these potentials were $-1.57 \pm-0.22 \mathrm{mV}(n=6$ crickets) in AN1 and $-0.71 \pm-0.13 \mathrm{mV}$ in $\mathrm{AN} 2(n=6$ crickets). If the ears of silently stridulating crickets were removed, the hyperpolarization was still present but had a lower amplitude both in AN1 $(-0.39 \mathrm{mV}, n=1)$ and AN2 $(-0.40 \pm$ $0.05 \mathrm{mV}, n=4)$. This is probably attributable to the removal of any spontaneously firing auditory afferents that would normally depolarize AN1 and AN2. With this depolarizing input removed, the membrane potential would return to its, slightly more negative, resting value and the hyperpolarization would appear to be smaller.

In $30 \%$ of experiments, EPSPs that sometimes generated spikes were recorded in AN1 $(n=3)$ and AN2 $(n=5)$ during

Ai और्थर
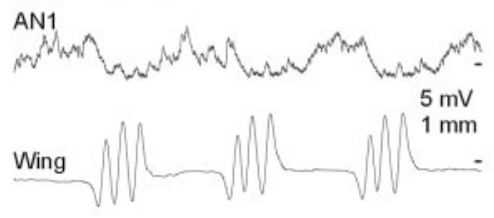

Sound
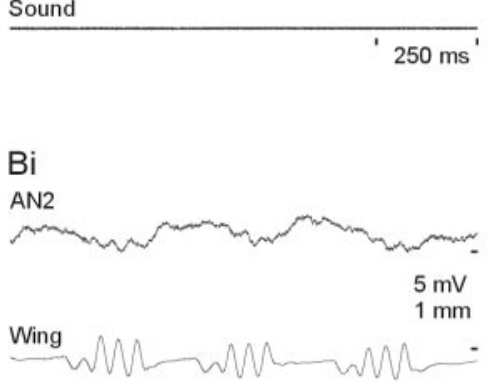

Sound

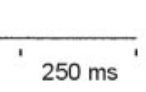

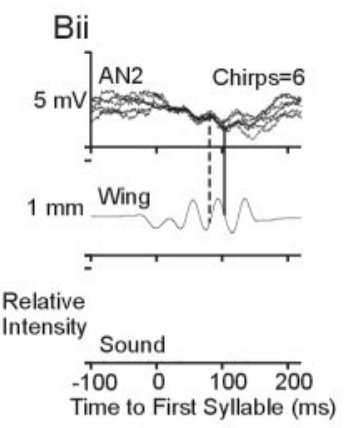

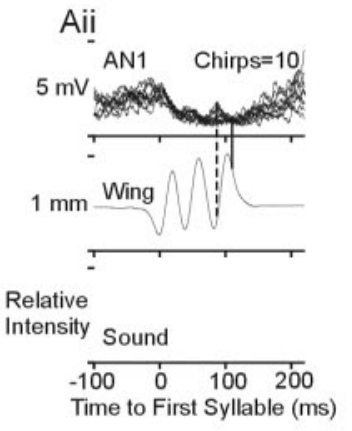

Figure 3. Low-amplitude hyperpolarizations were normally observed in both AN1 (Ai) and AN2 (Bi) during silent one-winged chirps. Superpositions of AN1 (Aii) and AN2 (Bii) (top traces), triggered by the onset of the wing movement (bottom traces), demonstrate the time course of the hyperpolarizations in relation to the average wing movement. They began just after the start of the closing wing movements, indicted by the dashed lines, and reached a maximum during the consecutive wing opening movements, indicated by the solid line. For additional details see Figure 2.

silent stridulation (Fig. $4 A i, B i$ ). On average, the EPSPs reached $5.48 \pm 2.18 \mathrm{mV}(n=3)$ in AN1 and $1.76 \pm 0.42 \mathrm{mV}(n=5)$ in AN2. The phase of the EPSP varied from animal to animal, but generally they started in phase with wing closing and reached a maximum during the consecutive wing opening (Fig. 4Aii,Bii). EPSPs were normally recorded during chirps with highamplitude wing movement. They could also be elicited by manually moving the right wing of intact $\mathrm{AN} 1(n=2)$ and $\mathrm{AN} 2(n=$ $4)$, and deafened AN1 $(n=1)$ and AN2 $(n=2)$ (Fig. 5), crickets. It is not known which sense organ mediates this excitation.

Therefore, the responses of AN1 and AN2 during sonorous stridulation are the result of a mix of inputs from reafferent sound and wing movement and a low-amplitude hyperpolarization. To determine whether the hyperpolarization was centrally generated or the result of nonauditory sensory feedback, we recorded the responses of AN1 and AN2 in crickets with their thoracic or thoracic and abdominal ganglia isolated. Even under these circumstances, the isolated CNS generated the motor output for stridulation. Because this type of stridulation did not involve any movement or sensory feedback it was termed fictive stridulation.

\section{Responses of AN1 and AN2 during fictive stridulation}

AN1 and AN2 rarely spiked during fictive stridulation (Fig. $6 A i, B i)$. Low-amplitude hyperpolarizing potentials were recorded in phase with the fictive chirps in four of five recordings of AN1 and in four of five recordings of AN2 (Fig. 6Aii,Bii). They increased in size during the chirp and reached an average maximum amplitude of $-2.2 \pm 0.73 \mathrm{mV}$ ( $n=4$ crickets) in AN1 and $-0.65 \pm 0.05 \mathrm{mV}$ ( $n=4$ crickets $)$ in AN2. Because of the delay between motor nerve activity and wing movement, the hyperpolarizations occurred slightly later during fictive chirps than dur- 

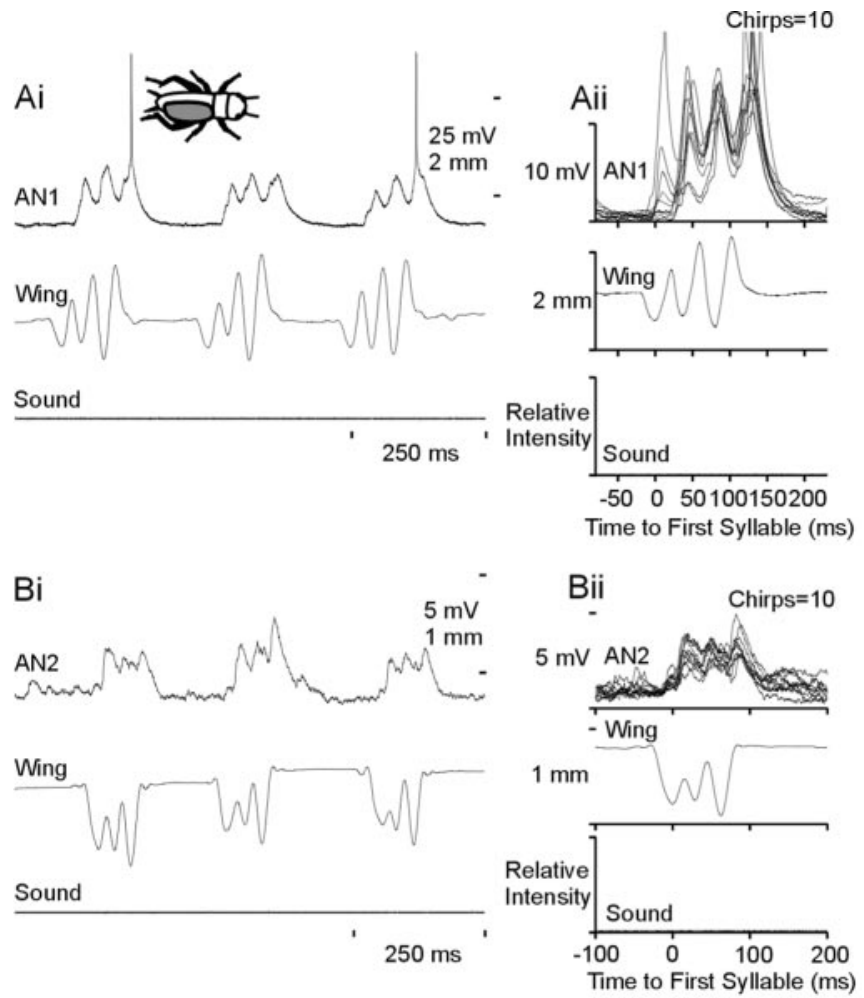

Figure 4. $A i, B i$, Depolarizations were sometimes observed in recordings of AN1 and AN2 during silent one-winged stridulation. Superimposed recordings of AN1 (Aii) and AN2 (Bii) together with the averaged wing movement and sound demonstrate the timing of the depolarizations in relation to the wing movement. The timing varied from animal to animal. In general, the depolarizations started during wing closing and peaked at the transition from closing to opening. The spikes have been truncated in the superpositions. For additional details see Figure 2.

ing silent chirps. In one of five crickets, in both AN1 and AN2, the membrane potential remained unchanged during fictive chirps. Depolarizing potentials were never recorded during fictive chirps in either neuron. With the cricket's ears removed, hyperpolarizing potentials were recorded during fictive chirps in one recording of AN1 $(-0.28 \mathrm{mV})$ and in one recording of AN2 $(-1.0 \mathrm{mV})$. Therefore, based on this limited result it appears that the hyperpolarizations were the result of a centrally generated corollary discharge.

\section{Responses of AN1 and AN2 to acoustic stimulation during stridulation}

The corollary discharge mediates presynaptic inhibition of auditory afferent terminals (Poulet and Hedwig, 2002, 2003) alongside the hyperpolarizations recorded in AN1 and AN2. To examine the combined effect of these inhibitory inputs on the responses of AN1 and AN2 to externally generated, or exafferent (von Holst and Mittelstaedt, 1950), sound stimuli, we presented silently and fictively stridulating crickets with trains of acoustic stimuli. The stimuli had a short period $(15 \mathrm{msec})$ and duration $(8$ $\mathrm{msec}$ ) to present them at all phases of the wing movement underlying stridulation.

AN1 and AN2 spiked consistently to the acoustic stimuli when the cricket was at rest and during chirp intervals but their auditory responses were inhibited during silent chirps (Figs. 7Ai,Bi). The inhibition began at the start of wing closing (Fig. 7Aii,Bii, dashed lines) and lasted throughout the chirp. Inhibition was present even in the cases in which EPSPs were generated during

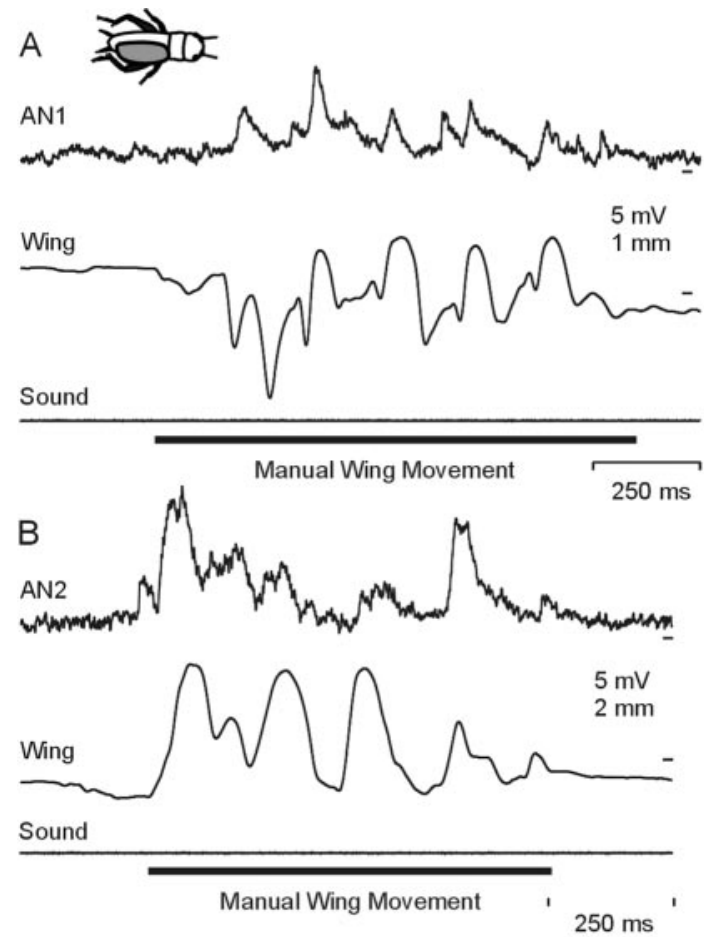

Figure 5. Response of AN1 and AN2 to wing movement in deafened crickets. EPSPs were elicited in AN1 $(A)$ and AN2 $(B)$ during manual wing movement in deafened crickets. For additional details see Figure 2 .

the silent chirps. EPSPs, which occasionally generated spikes, were recorded at the transition between wing opening and closing (Fig. 7Ai,Bi, asterisks). Wing movement and/or the increased afferent input during those phases of the chirp in which the amplitude of the inhibitory inputs to the afferents and interneurons was decreased may have mediated the EPSPs.

During fictive chirps, both neurons showed similar effects to those recorded during silent stridulation (Fig. 8A,B). However, the amplitude of the EPSPs compared with those recorded during silent chirps was reduced. Therefore, the inhibition mediated by the corollary discharge (hyperpolarizations in AN1 and AN2 and presynaptic inhibition of auditory afferents) suppresses the responses of AN1 and AN2 to sound during the chirps.

\section{The impact of the inhibition on the processing of sound patterns similar to cricket song}

We then examined the strength of the corollary discharge inhibition on auditory processing in silently stridulating crickets presented with more natural stimuli. When recording from AN1 (Fig. 9A), we presented the cricket with acoustic stimuli that mimicked cricket song $(4.5 \mathrm{kHz}, 100 \mathrm{~dB}$ SPL, 21 msec duration, 21 msec period); when recording from AN2 (Fig. 9B) we used $12.5 \mathrm{kHz}$ sound pulses (21 msec duration, $250 \mathrm{msec}$ period). Because AN2 was normally inhibited by $4.5 \mathrm{kHz}$ stimuli, we did not examine its response at this frequency. At rest and during the chirp intervals, AN1 responded with a burst of $\sim 5$ spikes at $337 \pm$ $52 \mathrm{~Hz}$ ( $n=6$ crickets). During silent chirps the responses of the same neurons were reduced to bursts of only 1-2 spikes at $97 \pm$ $29 \mathrm{~Hz}$, which was significantly lower than the response during the chirp interval (two-tailed paired $t$ test; $p<0.001 ; t=-7.24$; $\mathrm{df}=$ 5). AN2 responded vigorously to the $12.5 \mathrm{kHz}$ acoustic stimuli when at rest and during the chirp intervals with bursts of $\sim 15$ spikes at a rate of $353 \pm 28 \mathrm{~Hz}(n=9$ crickets $)$. The same neurons 

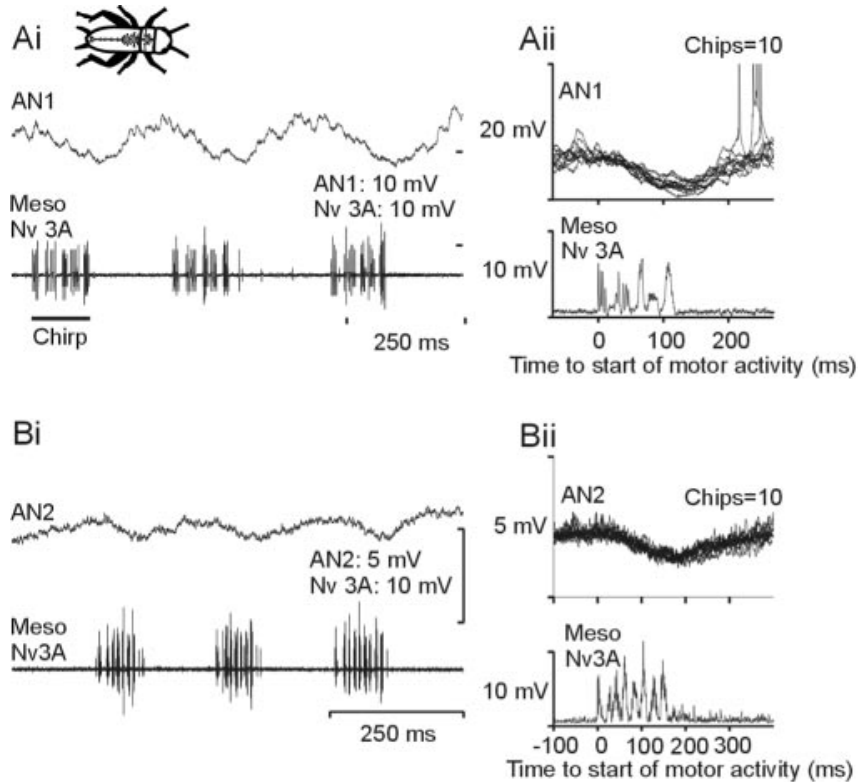

Figure 6. Activity of AN1 and AN2 during fictive stridulation. Low-amplitude hyperpolarizations were recorded in AN1 ( $\mathrm{Ai}$ ) and AN2 (Bi) during the fictive chirps with the similar amplitude and timing as in silently stridulating crickets. Fictive chirps are indicated by thoracic motor activity. Aii, Bii, Superimposed traces of neuron recordings (top) show the timing of the PADs and IPSPs in relation to the averaged, rectified mesothoracic nerve 3 A recording (bottom). Meso Nv $3 \mathrm{~A}$, Extracellular nerve recording with several units of opener and closer motor neuron activity. For additional details see Figure 2 .

responded with bursts of only three spikes at $108 \pm 18 \mathrm{~Hz}$ during the silent chirps, which was significantly lower than the response during the chirp intervals (two-tailed paired $t$ test; $p<0.001 ; t=$ 10.33; $\mathrm{df}=8$ ).

The responses of AN1 to $100 \mathrm{~dB}$ SPL sound pulses varied depending on the phase of stimulation. For example, compare the responses to each of the four stimuli presented during the silent chirp in Figure 9A. To examine this difference in more detail, we plotted the maximum spike frequency of the response to each acoustic stimulus presented during silent stridulation against wing movement and then calculated the average response of AN1 (Fig. 10, gray line). During the chirp interval AN1 responded with bursts of spikes (in this example at $\sim 260 \mathrm{~Hz}$ ). The response of AN1 was maximally reduced (in this example to $\sim 10$ $\mathrm{Hz}$ ) during the opening wing movements (Fig. 10, gray bars), which coincides with the maximum response to self-generated sound (Fig. 2Aii). At the transition between wing opening to closing the inhibition was weaker and AN1 generated bursts of spikes. Therefore, the inhibition is timed to reduce reafferent auditory stimulation of AN1.

\section{Response of AN1 to sound patterns mimicking cricket song}

We have shown previously that the stimulation of ON1 in the male cricket, induces a burst of spikes followed by a long-lasting hyperpolarization (Poulet and Hedwig, 2002, 2003). A similar effect was present in AN1. A long-lasting hyperpolarization always followed a burst of spikes in response to a 1 sec sequence of $4.5 \mathrm{kHz}$ stimuli (Fig. 11A). To examine the effect of the hyperpolarization, we presented resting crickets with stimuli that mimicked their own song and examined their response to quieter test stimuli $(n=4)$. The control series of test pulses ( $80 \mathrm{~dB}$ SPL) on average elicited bursts of $5.6 \pm 0.7$ spikes with an average maximum spike frequency of $294 \pm 37 \mathrm{~Hz}$ (Fig. 11 B,C). Stimuli mim-
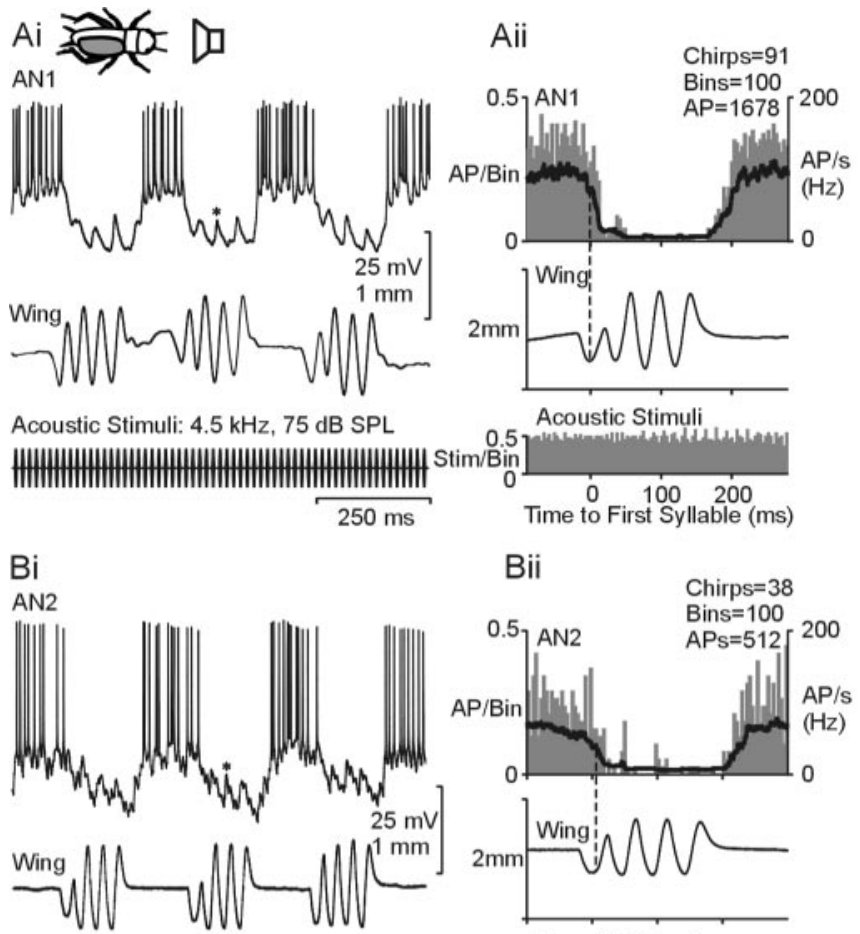

Acoustic Stimuli: $12.5 \mathrm{kHz}, 75 \mathrm{~dB}$ SPL
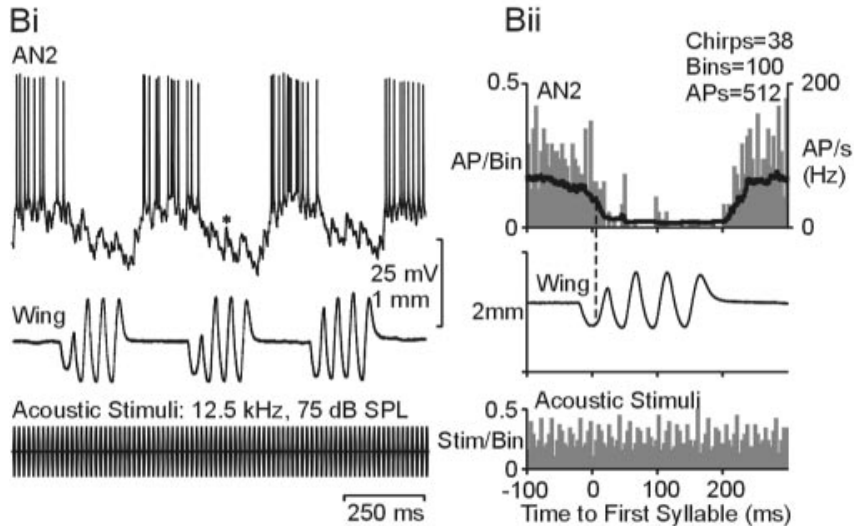

Figure 7. Response of AN1 and AN2 to acoustic stimuli during silent stridulation. Ai, The spiking response of AN1 to a train of sound pulses ( $4.5 \mathrm{kHz}, 75 \mathrm{dBSPL}, 7$ msec duration, $15 \mathrm{msec}$ interval) is inhibited during the chirps (asterisk). Aii, Quantitative analysis showing the PST histogram with the superimposed spike frequency (top), the averaged wing movement (middle), and the distribution of the sound stimuli (bottom) shows a clear inhibition of the response of AN1 during silent chirps. The inhibition began at the start of the first wing closing, as indicated by the dashed line. Bi, AN2 responded to a train of sound stimuli $(12.5 \mathrm{kHz}, 75 \mathrm{~dB} \mathrm{SPL}, 7$ msec duration, 15 msec interval) during the chirp intervals, but its response was inhibited during the chirps. EPSPs were recorded during the chirp (asterisk), and occasionally spikes were recorded on top of the larger EPSPs during the transition between closing and opening wing movement. Bii, Again the PST histogram and the average spike frequency of the response of AN2 show a clear reduction during the chirp that began at the start of wing closing, as indicated by the dashed line. The bottom PST histogram in Aii and Bii shows that the sound stimuli were evenly distributed throughout the chirp and the chirp interval. For additional details see Figure 2.

icking calling song were then presented to the cricket before the $80 \mathrm{~dB}$ SPL test stimuli. The numbers of spikes per burst were then reduced by $21 \%$, to $4.4 \pm 0.7$ spikes, which was accompanied by an $8 \%$ reduction in maximum spike frequency to $270 \pm 38 \mathrm{~Hz}$ (Fig. $11 \mathrm{~B}, C$ ). The reduction was greatest just after the $100 \mathrm{~dB}$ SPL chirp and recovered after 100-200 msec (Fig. 11C). Therefore, spiking during sonorous stridulation could desensitize AN1 and reduce its sensitivity to quieter external sounds immediately after the animal's own chirps.

\section{Discussion}

We have demonstrated previously that the cricket's tympanic nerve and tympanic membrane respond fully to self-generated sounds (Poulet and Hedwig, 2001). The intense input from the peripheral auditory system during stridulation could disrupt the processing of important external sounds. However, behavioral experiments have shown that crickets maintain auditory responsiveness during stridulation (Heiligenberg, 1969; Jones and Dambach, 1973). In this study, we examined the responses of two 

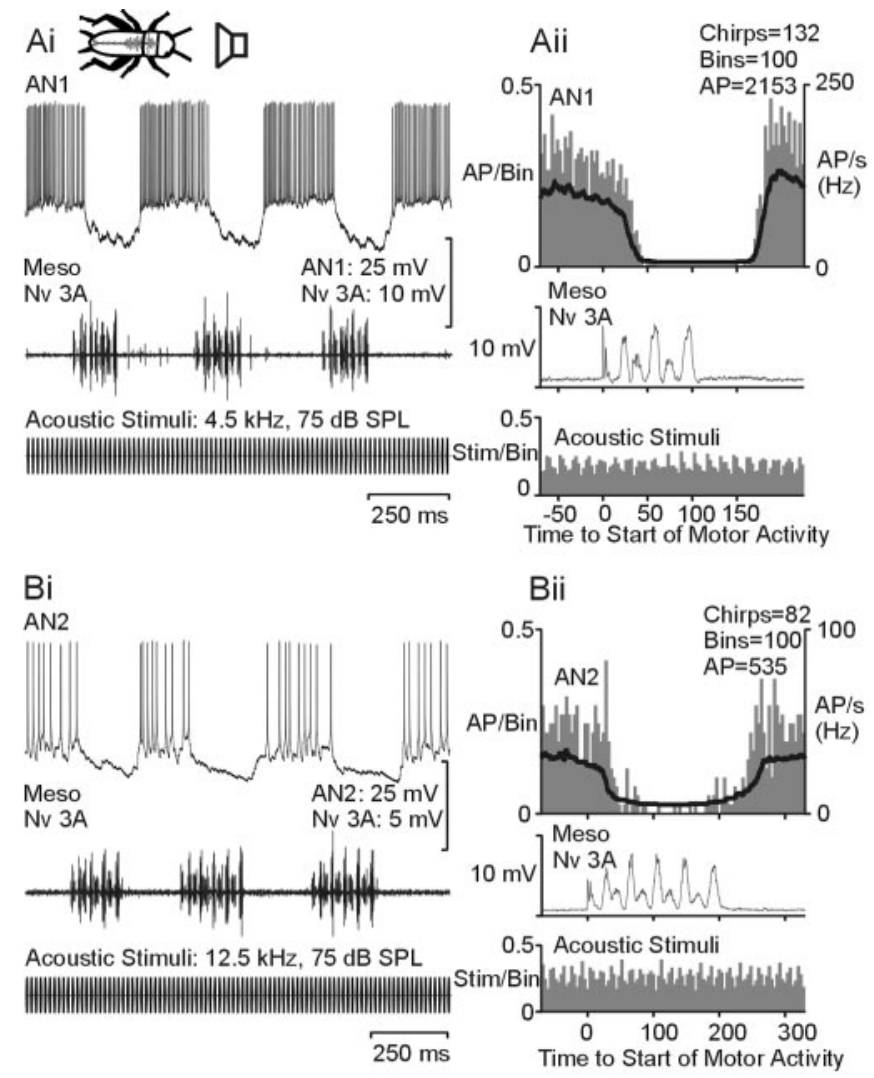

Figure 8. Auditory responses to acoustic stimulation during fictive singing. Ai, AN1 spiked to the sequence of $4.5 \mathrm{kHz}, 75 \mathrm{~dB}$ SPL acoustic stimuli during the chirp interval, but was inhibited during the chirp. Aii, Inhibition during the chirp was clearly demonstrated by the PST histogram, with an overlaid average spike rate. $B i$, The spiking response of AN2 to $12.5 \mathrm{kHz}$ acoustic stimuli was inhibited during the fictive chirps but not during the chirp intervals. Bii, The PST histogram and overlaid average spike rate (top) of the response of AN2 to acoustic stimuli (bottom) show an inhibition of the response of AN2 during the fictive chirp, as indicated by the rectified and averaged motor activity (middle). For additional details see Figure 2.

identified ascending auditory neurons (AN1 and AN2) to selfgenerated and external sounds during stridulation. We demonstrate that their responses to self-generated sound are reduced by a centrally generated corollary discharge.

\section{Frequency tuning of AN1 and AN2}

AN1 and AN2 are two identified ascending auditory interneurons that project from the prothoracic ganglion to the brain. AN2 is inhibited or weakly excited by $4.5 \mathrm{kHz}$ and responds best to $12.5 \mathrm{kHz}$ (Wohlers and Huber, 1978, 1982; Nolen and Hoy, 1987), whereas AN1 responds best to $4.5 \mathrm{kHz}$ acoustic stimuli (Boyan, 1980; Wohlers and Huber, 1982; Stumpner et al., 1995). In this study, we show that $12.5 \mathrm{kHz}$ stimuli elicit IPSPs in AN1 that inhibit spiking (Fig. 1). Postsynaptic inhibition to highfrequency stimuli has been recorded in the homolog neuron L1 of AN1 in Acheta domesticus (Stumpner et al., 1995). Frequencydependent inhibition should sharpen the frequency selectivity of AN1, but neither the neurons that mediate this inhibition nor the behavioral significance is fully understood (Stumpner et al., 1995).

\section{Inputs to AN1 and AN2 during stridulation}

AN1 spiked whereas AN2 received high-amplitude IPSPs during sonorous chirps (Fig. 2). During silent stridulation, only lowamplitude hyperpolarization or depolarizations were recorded in
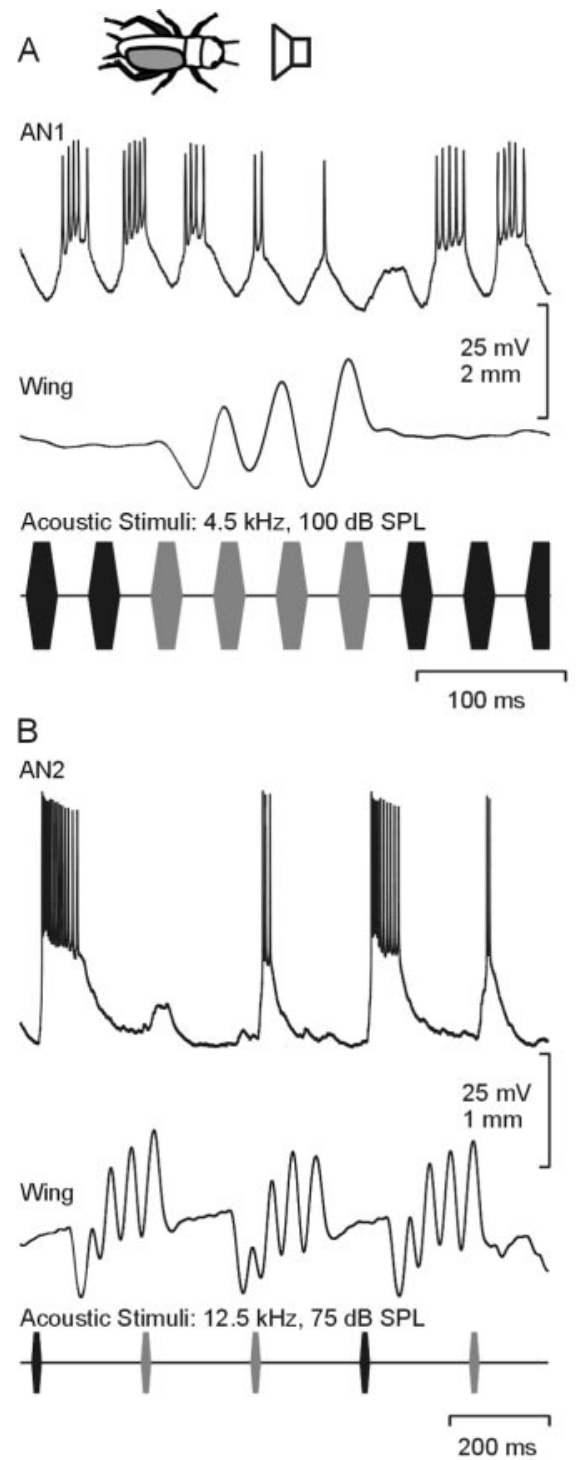

Figure 9. Effectiveness of the centrally generated inhibition during silent stridulation. $A$, AN1 responded to $100 \mathrm{~dB}$ SPL, $4.5 \mathrm{kHz}$ acoustic stimuli during the chirp intervals (black) with bursts of spikes. The strength of response was reduced during the chirps (gray). B, AN2 responded to $12.5 \mathrm{kHz}, 75 \mathrm{~dB}$ SPL acoustic stimuli with bursts of spikes during the chirp interval (black). The response was reduced during the chirp (gray). For additional details see Figure 2.

AN1 and AN2 (Figs. 3, 4). Stimuli at the carrier frequency of cricket calling song $(4.5 \mathrm{kHz})$ elicit very similar responses in AN1 and AN2. Therefore, the reactions during sonorous chirps can be explained as a response to self-generated sound.

Two other inputs to AN1 and AN2 were identified during silent stridulation. In 70\% of experiments low-amplitude hyperpolarizing potentials were observed in AN1 and AN2 during silent (Fig. 3) and fictive (Fig. 6) chirps. Their timing was very similar to the IPSPs recorded in ON1 (Poulet and Hedwig, 2002, 2003); both started in phase with wing closing and reached a maximum during the consecutive wing opening, but were of much lower in amplitude. What appear to be hyperpolarizations could in fact be a reduction in EPSPs reaching AN1 and AN2 because of the presynaptic inhibition of spontaneously firing afferents during the chirps. However, hyperpolarizations were also recorded in silently and even in fictively stridulating crickets with their ears removed. Therefore, we conclude that they are generated within the nervous system and probably are the result of the 


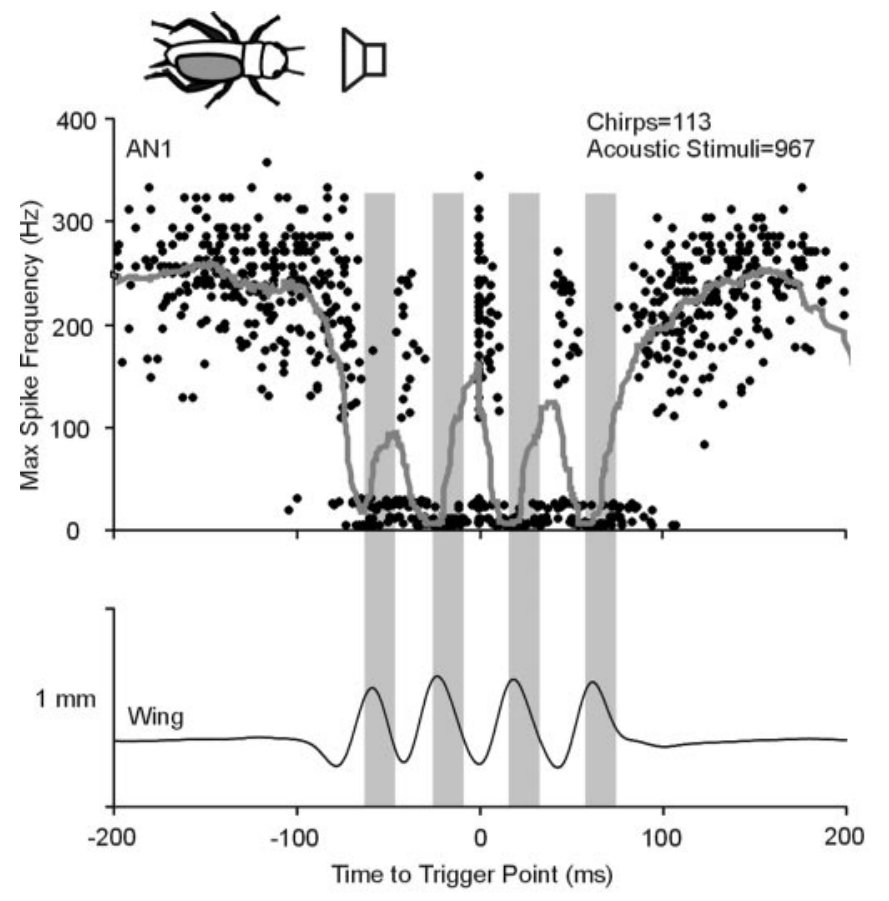

Figure 10. Timing of the centrally generated inhibition in AN1. The maximum spike frequency of AN1 to individual $4.5 \mathrm{kHz}, 100 \mathrm{~dB}$ SPL stimuli presented during silent stridulation was plotted as a dot against the average wing movement. The gray line represents the average spike frequency. The close temporal relationship of responses near the temporal reference point at 0 sec is attributable to the analysis procedure. Gray bars show that the timing of the maximum response reduction during silent singing coincides with the opening wing movement, which is the time AN1 would respond to self-generated sound. For additional details see Figure 2.

same corollary discharge that postsynaptically inhibits ON1 and presynaptically inhibits the terminals of the auditory afferents.

In $30 \%$ of recordings of $\mathrm{AN} 1$ and $\mathrm{AN} 2$, depolarizing potentials were recorded during silent chirps that occasionally elicited spikes (Fig. 4). Three observations led us to conclude that they were generated by reafferent feedback via an unidentified sensory pathway activated by wing movement: (1) chirps that caused depolarizations generally were produced by high-amplitude wing movements; (2) depolarizing potentials were never recorded during fictive stridulation with all sensory feedback removed; and (3) depolarizing potentials were elicited by manual wing movement in deafened crickets (Fig. 4). Auditory interneurons in the cricket, e.g., ON1 (Wiese, 1981; our unpublished observations), and in the grasshopper, e.g., the G-neuron (Bickmeyer et al., 1992), receive inputs from more than one sense organ.

\section{Responses to external sound during stridulation}

AN1 and AN2 responded to acoustic stimulation during chirp intervals, but their responses were inhibited during silent and fictive chirps (Figs. 7, 8). This was attributable to presynaptic inhibition of the auditory afferent terminals and the lowamplitude hyperpolarizations in AN1 and AN2. The strength of the combined inhibition was tested by playing test stimuli at the best frequency of the neuron to silently stridulating crickets. AN2 responded vigorously during the chirp interval (Fig. 9), but its response was reduced by $65 \%$ during the chirps. The response of AN1 to stimuli that mimicked cricket song $(4.5 \mathrm{kHz}, 100 \mathrm{~dB})$ was reduced by $71 \%$ during silent chirps (Fig. 9). The percent reduction in response to external stimuli was very similar in AN1 (71\%) and AN2 (65\%) to that recorded in ON1 (67\%) (Poulet and Hedwig, 2002, 2003). The response in resting crickets was
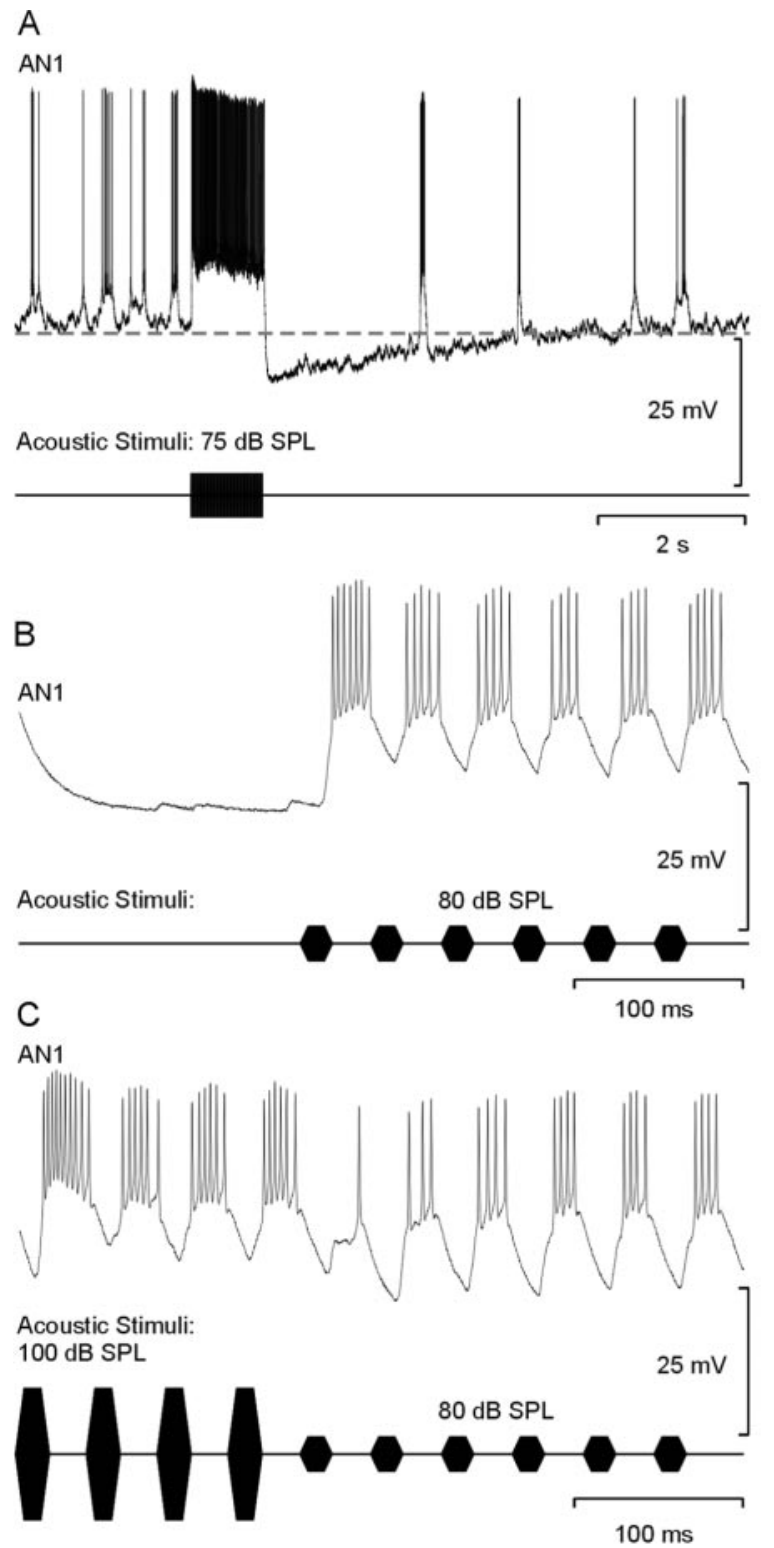

Figure 11. A, A long-lasting hyperpolarization that followed spiking of AN1 in response to a train of $4.5 \mathrm{kHz}, 75 \mathrm{~dB}$ SPL stimuli. The dashed line indicates the resting membrane potential before acoustic stimulation. $B$, AN1 responded vigorously to a series of $4.5 \mathrm{kHz}, 80 \mathrm{~dB}$ SPL stimuli. C, The response of AN1 to the $80 \mathrm{~dB}$ SPL stimuli was slightly reduced if they were preceded by $100 \mathrm{~dB}$ SPL chirps. For additional details see Figure 2 .

higher than the response to self-generated sounds, which confirmed a reduction in response to self-generated sounds; however, the response during the silent chirps was lower than the response to self-generated sound. This effect was also recorded in ON1 (Poulet and Hedwig, 2002, 2003) and is probably attributable to differences in the stimulus design compared with natural sounds.

The presynaptic afferent depolarizations (PADs) in the afferents (Poulet and Hedwig, 2002, 2003) and the hyperpolarizations in the ascending neurons (Figs. 3, 6) and ON1 begin just after the start of wing closing and reach a maximum just after the transition from wing closing to opening. Therefore, we examined individual responses to $4.5 \mathrm{kHz}, 100 \mathrm{~dB}$ SPL acoustic stimuli presented throughout silent chirps and compared it with the activity of AN1 during sonorous stridulation (Fig. 10). Figure 10 shows 
that the maximum reduction in response coincides with the time that AN1 would normally respond to self-generated sounds. As in $\mathrm{ON} 1$, the corollary discharge is exactly timed to inhibit the response of AN1 to self-generated sound.

\section{Biological significance of the corollary discharge}

In this study, we recorded a long-lasting hyperpolarization in AN1 that followed a spiking response to a series of $4.5 \mathrm{kHz}$ stimuli (Fig. 11). A very similar effect has also been recorded in ON1 (Pollack, 1988; Sobel and Tank, 1994; Römer and Krusch, 2000; Poulet and Hedwig, 2002, 2003). It causes ON1 to respond to the louder of two acoustic stimuli (Pollack, 1988; Römer and Krusch, 2000; Poulet and Hedwig, 2002, 2003) and is thought to be a cellular property of ON1 (Sobel and Tank, 1994, Poulet and Hedwig, 2002, 2003). We suspect that the same is true for the hyperpolarization recorded in AN1. The response of AN1 to $80 \mathrm{~dB}$ SPL test stimuli was reduced if it was preceded by a $100 \mathrm{~dB}$ SPL chirp (Fig. 11). However, in comparison with ON1 the reduction of the response of AN1 was much weaker. This could be because the hyperpolarization has less effect in AN1. It may also be because the response of AN1 saturates at a lower intensity than that of ON1 (Stumpner et al., 1995), in which case there would be a smaller difference in the spiking response to 100 and $80 \mathrm{~dB}$ SPL stimuli in AN1 than in ON1. Corollary discharge inhibition during stridulation will reduce the amount of spiking in AN1 and should counteract any desensitizing effects of the hyperpolarization. This will increase the sensitivity of AN1 to external sounds during the chirp interval.

Spiking in AN2 during flight elicits escape behavior in Teleogryllus (Nolen and Hoy, 1983). Therefore, it is not surprising that alongside the corollary discharge inhibition an additional frequency-dependent inhibition exists at the carrier frequency of calling song. The effectiveness of the corollary discharge at inhibiting responses to exafferent sound was similar in AN1 and ON1. This was surprising because the amplitude of the hyperpolarizations mediated by the corollary discharge was much smaller in AN1 and AN2 compared with those recorded in ON1. This may be because the ionic reversal potential of the inhibitory synaptic input is closer to the resting membrane potential of AN1 and AN2 than to that of ON1, in which case it may have a similar effect but be more difficult to observe. However, it could also be because there is less need for additional postsynaptic inhibition of AN1 and AN2 alongside the presynaptic inhibition during stridulation. For example, the desensitizing effect of the long-lasting hyperpolarization that follows spiking is weaker in AN1 than in ON1; thus, AN1 should be more responsive than ON1 to external sounds during sonorous stridulation, in which case there would be less need to inhibit AN1. In cases in which EPSPs were recorded in AN1 and AN2 during silent stridulation, their response to exafferent sound was still inhibited during the chirps. This implies that presynaptic inhibition on its own is an effective mechanism to selectively cancel auditory input to AN1 and AN2 during stridulation. Perhaps it is more effective in AN1 and AN2 than in ON1, which therefore requires an additional postsynaptic inhibition. Experiments that separate the relative contributions of the presynaptic and postsynaptic inhibition to the neural responses of the auditory interneurons would be technically difficult if, as we suspect, both types of inhibition have the same source.

\section{Future experiments}

Studies made on vocalizing vertebrates have also indicated a reduction in neural activity during sound production (Suga and
Schlegel, 1972; Suga and Shimozawa, 1974; Schuller 1979; McCasland and Konishi, 1981; Müller-Preuss and Ploog, 1981; Creutzfeldt et al., 1989; Metzner, 1989, 1993; Kirzinger and Jürgens, 1991; Numminen et al., 1999; for a wider discussion, see Poulet and Hedwig, 2003) but were not able to identify the source of the inhibition. In the cricket, the corollary discharge inhibits AN1 and AN2 at the same phases of the chirp as the afferents and ON1; therefore, it is likely to have the same source. We aim to identify the neuron(s) responsible for the inhibition, which we suspect will be part of the pattern-generating network for stridulation. The origin of the excitatory input to AN1 and AN2 is not yet known. A first step would be to identify exactly which sense organ is responsible for this input. We suspect that the hyperpolarization after spiking in AN1 is a cellular property of AN1, as is thought to be the case in ON1 (Sobel and Tank, 1994; Poulet and Hedwig, 2002, 2003), but more experiments are required to fully characterize its source and understand its role in auditory processing in resting crickets.

\section{References}

Bell CC (1981) An efference copy which is modified by reafferent input. Science 214:450-453.

Bell CC (1982) Properties of a modifiable efference copy in an electric fish. J Neurophysiol 47:1043-1056.

Bickmeyer U, Kalmring K, Halex H, Mucke A (1992) The bimodal auditoryvibratory system of the thoracic ventral nerve cord in Locusta migratoria (Acrididae, Locustinae, Oedipodini). J Exp Zool 264:381-394.

Blakemore S-J, Wolpert DW, Frith CD (1998) Central cancellation of selfproduced tickle sensation. Nat Neurosci 1:635-640.

Bodznick D, Montgomery JC, Carey M (1999) Adaptive mechanisms in the elasmobranch hindbrain. J Exp Biol 202:1357-1364.

Borg E, Counter S (1989) The middle-ear muscles. Sci Am 261:62-68.

Boyan GS (1980) Auditory neurones in the brain of the cricket Gryllus bimaculatus (De Geer). J Comp Physiol [A] 140:81-93.

Creutzfeldt O, Ojemann G, Lettich E (1989) Neuronal activity in the human lateral temporal lobe: II. Responses to the subjects own voice. Exp Brain Res 77:476-489.

El Manira A, Tegnér J, Grillner S (1996) Locomotor-related presynaptic modulation of primary afferents in the lamprey. Eur J Neurosci 9:696-705.

Gossard J-P, Cabelguen J-M, Rossignol S (1991) An intracellular study of muscle primary afferents during fictive locomotion in the cat. J Neurophysiol 65:914-926.

Guthrie BL, Porter JD, Sparks DL (1983) Corollary discharge provides accurate eye position information to the oculomotor system. Science 221:1193-1195.

Heiligenberg W (1969) The effect of stimulus chirps on a cricket's chirping. Z Vergl Physiol 65:70-97.

Hennig RM (1988) Ascending auditory interneurons in the cricket Teleogryllus commodus (Walker): comparative physiology and direct connections with afferents. J Comp Physiol [A] 163:135-143.

Hennig RM, Weber T, Huber F, Kleindienst H-U, Moore TE, Popov AV (1994) Auditory threshold change in singing cicadas. J Exp Biol 187:45-55.

Jones MDR, Dambach M (1973) Response to sound in crickets without tympanal organs (Gryllus campestris L.). J Comp Physiol [A] 87:89-98.

Kirzinger A, Jürgens U (1991) Vocalization-correlated single-unit activity in the brain stem of the monkey. Exp Brain Res 84:545-560.

Knepper M, Hedwig B (1997) NEUROLAB, a PC-program for the processing of neurobiological data. Comput Methods Programs Biomed 52:75-77.

McCasland JS, Konishi M (1981) Interaction between auditory and motor activities in an avian song control nucleus. Proc Natl Acad Sci USA 78:7815-7819.

Metzner W (1989) A possible neuronal basis for Doppler-shift compensation in echo-locating horseshoe bats. Nature 341:529-532.

Metzner W (1993) An audio-vocal interface in echolocating horseshoe bats. J Neurosci 13:1899-1915.

Murphey RK, Palka J (1974) Efferent control of cricket giant fibres. Nature 248:249-251.

Müller-Preuss P, Ploog D (1981) Inhibition of auditory cortical neurons during phonation. Brain Res 215:61-76. 
Narins PM (1992) Reduction of tympanic membrane displacement during vocalization of the arboreal tree frog, Eleutherodactylus coqui. J Acoust Soc Am 91:3551-3557.

Nolen TG, Hoy RR (1983) Initiation of behaviour by single neurons: the role of behavioural context. Science 226:992-994.

Nolen TG, Hoy RR (1987) Postsynaptic inhibition mediates high-frequency selectivity in the cricket Teleogryllus oceanicus: implications for flight pyhonotaxis behavior. J Neurosci 7:2081-2096.

Numminen J, Salmelin R, Hari R (1999) Subject's own speech reduces reactivity of the human auditory cortex. Neurosci Lett 265:119-122.

Otto D (1978) Änderungen von Gesangsparametern bei der Grille (Gryllus campestris L.) nach Injektion von Pharmaka ins Gehirn. Vh Dt Zool Ges 245.

Pollack GS (1988) Selective attention in an insect auditory neuron. J Neurosci 8:2635-2639.

Poulet JFA, Hedwig B (2001) Tympanic membrane oscillations and auditory receptor activity in the stridulating cricket Gryllus bimaculatus. J Exp Biol 204:1281-1293.

Poulet JFA, Hedwig B (2002) A corollary discharge maintains auditory sensitivity during sound production. Nature 418:872-876.

Poulet JFA, Hedwig B (2003) A corollary discharge mechanism modulates central auditory processing in singing crickets. J Neurophysiol 89:1528-1540.

Römer H, Krusch M (2000) A gain-control mechanism for processing of chorus sounds in the afferent auditory pathway of the bushcricket Tettigonia viridissima (Orthoptera; Tettigoniidae). J Exp Biol 186:181-191.

Schuller G (1979) Vocalization influences auditory processing in collicular neurons of the CF-FM-bat, Rhinolophus ferrumequinum. J Comp Physiol [A] 132:39-46.

Sillar KT, Roberts A (1988) A neuronal mechanism for sensory gating during locomotion in a vertebrate. Nature 331:262-265.

Sillar KT, Skorupski P (1986) Central input to primary afferent neurons in crayfish, Pacifastacus leniusculus, is correlated with rhythmic motor output of thoracic ganglia. J Neurophysiol 55:678-688.
Sobel EC, Tank DW (1994) In vivo $\mathrm{Ca}^{2+}$ dynamics in a cricket auditory neuron: an example of chemical computation. Science 263:823-826.

Sperry RW (1950) Neural basis of the spontaneous optokinetic response produced by visual inversion. J Comp Physiol Psych 43:482-489.

Stumpner A, Atkins G, Stout JF (1995) Processing of unilateral and bilateral auditory inputs by the $\mathrm{ON} 1$ and $\mathrm{L} 1$ interneurons of the cricket Acheta domesticus and comparison to other cricket species. J Comp Physiol [A] 177:379-388.

Suga N, Jen P (1975) Peripheral control of acoustic signals in the auditory system of echolocating bats. J Exp Biol 62:277-311.

Suga N, Schlegel P (1972) Neural attenuation of responses to emitted sounds in echolocating bats. Science 177:82-84.

Suga N, Shimozawa T (1974) Site of neural attenuation of responses to selfvocalized sounds in echolocating bats. Science 183:1211-1213.

von Holst E, Mittelstaedt H (1950) Das Reafferenzprinzip: Wechselwirkungen Zwischen Zentralnervensystem und Peripherie. Naturwissenschaften 37:464-476.

Wenzel B, Hedwig B (1999) Neurochemical control of cricket stridulation revealed by pharmacological injections into the brain. J Exp Biol 202:2203-2216.

Wiese K (1981) Influence of vibration on cricket hearing: interaction of low frequency vibration and acoustic stimuli in the Omega neuron. J Comp Physiol [A] 143:135-142.

Wohlers DW, Huber F (1978) Intracellular recording and staining of cricket auditory interneurons (Gryllus campestris L., Gryllus bimaculatus DeGeer). J Comp Physiol [A] 127:11-28.

Wohlers DW, Huber F (1982) Processing of sound signals by six types of neurons in the prothoracic ganglion of the cricket, Gryllus campestris L. J Comp Physiol [A] 146:161-173.

Wolf H, Burrows M (1995) Proprioceptive sensory neurons of a locust leg receive rhythmic presynaptic inhibition during walking. J Neurosci 15:5623-5636.

Zaretsky M, Rowell CHF (1979) Saccadic suppression by corollary discharge in the locust. Nature 280:583-585. 